\title{
Epigenetics and expression of key genes associated with cardiac fibrosis: NLRP3, MMP2, MMP9, CCN2/CTGF, and AGT
}

\author{
Sruti Chandra ${ }^{\mathrm{a}}$, Kenneth C. Ehrlich ${ }^{\mathrm{b}}$, Michelle Lacey, ${ }^{\mathrm{c}, \mathrm{d}}$, \\ Carl Baribault $^{\mathrm{e}}$, Melanie Ehrlich ${ }^{\mathrm{b}, \mathrm{d}, \mathrm{f}_{*}}$
}

${ }^{a}$ Tulane Research Innovation for Arrhythmia Discoveries (TRIAD), Tulane

University School of Medicine, New Orleans, LA, USA

${ }^{b}$ Tulane Center for Biomedical Informatics and Genomics, Tulane University

Health Sciences Center, New Orleans, LA, USA

${ }^{c}$ Department of Mathematics, Tulane University, New Orleans, LA, USA

${ }^{d}$ Tulane Cancer Center, Tulane University Health Sciences Center, New Orleans, LA, USA

${ }^{e}$ Center for Research and Scientific Computing (CRSC), Tulane University Information Technology, New Orleans, LA, USA

${ }^{f}$ Hayward Genetics Center, Tulane University Health Sciences Center, New Orleans, LA, USA

*Corresponding Author

E-mail addresses: schandr1@tulane.edu (S. Chandra), kehrlich@tulane.edu (K.C. Ehrlich), mlacey1@tulane.edu (M. Lacey), cbaribault@ tulane.edu (C. Baribault), ehrlich@tulane.edu (M. Ehrlich).

Short title: Epigenetics of cardiac fibrosis genes

Keywords: Fibrosis; cardiovascular disease; inflammasomes; epigenetics; enhancers; DNA methylation 


\section{Abstract}

Excessive inflammatory signaling and pathological remodeling of the extracellular matrix are important contributors to cardiac fibrosis and involve major changes in gene expression. We examined the relationships between tissue-specific expression and the epigenetics of five genes involved in these pathways, NLRP3, MMP2, MMP9, CCN2/CTGF, and AGT. The proteins encoded by these genes play major fibrosis-related roles in inflammasome formation, degradation of extracellular matrix proteins and remodeling of the extracellular matrix and vasculature, autocrine regulation of fibrosis, or cell signaling. Our analyses showed that the first four of these genes had super-enhancers (unusually strong enhancer clusters) that correlate with their very high expression in monocytes, neutrophils, fibroblasts, or venous cells. Expression of the gene encoding miR-223, a micro-RNA that plays an important role in downregulating NLRP3 protein levels, is also probably driven by the super-enhancer in which it is embedded. Enhancer chromatin for all these genes was inside as well as outside the gene body. While $A G T$, which encodes precursors of angiotensin II, lacked a super-enhancer, its tissue-specific expression profile correlates with the tissue-specific enhancer chromatin extending into its distant silent gene neighbor (CAPN9). Tissue-specific peaks of DNA hypomethylation, open chromatin (DNaseI hypersensitivity), and transcription factor binding were detected in subregions of these super-enhancers/enhancers that are likely to be the main drivers of expression of their associated gene. We found that $C C N 2 / C T G F$ is co-expressed with its farupstream neighbor LINC01013, a noncoding RNA gene, specifically in vein endothelial cells (HUVEC) and chondrocytes. Evidence from chromatin looping profiling (Hi-C) suggests coregulation of these genes in HUVEC. Our findings indicate the importance of understanding the often-overlooked roles of enhancers and their hypomethylated, transcription factor-binding subregions in the regulation of expression of fibrosis-related genes in normal and fibrotic tissue. 


\section{Introduction}

Fibrotic disease is estimated to be responsible for almost half of the deaths in the US [1], and its far-reaching consequences extend to COVID-19's effects on disparate tissues and organs [2]. Cardiac fibrosis involves the remodeling of the heart accompanied by the excessive accumulation of extracellular matrix (ECM) proteins due to chronic stress, injury, systemic disease, or drugs $[3,4]$. Because the scar tissue impedes the ability of the heart to pump properly, cardiac fibrosis plays a causative role in many types of heart disease, including atrial fibrillation, heart failure, and impaired systolic and diastolic function. The main cell types that contribute to cardiac fibrosis include cardiac fibroblasts (which constitute $\sim 70 \%$ of the nuclei in the heart), myofibroblasts (which very highly express ECM proteins and are derived from cardiac fibroblasts or infiltrating fibrocytes), cardiomyocytes, cardiac endothelial cells, monocytes, neutrophils, and T cells $[4,5]$.

Epigenetics refers to the inherited changes from cell to cell that involve DNA and chromatin but do not alter the sequence of bases in DNA. Changes in epigenetics, usually histone or DNA modifications, and alterations in the composition and conformation of chromatin provide a kind of cellular memory. This memory is needed for differentiation and, to a lesser extent, for sustained responses to some types of physiological changes [6], including in the heart [7]. Epigenetics is involved in turning genes on or off, up- or down-modulating their levels of expression, or maintaining levels of transcription or transcription repression in normal and diseased tissues $[6,8]$. The importance of understanding the normal epigenetic modifications of cardiac fibrosis-associated genes is underscored by the involvement of differentiation in fibrosis, namely, transdifferentiation of fibroblasts and fibrocytes to myofibroblasts and inflammationassociated differentiation of monocytes to macrophages [9]. 
The critical role of epigenetic changes in cardiac fibrosis is beginning to be elucidated [7, 9-12]. For example, Williams et al. [3] examined the involvement of histone acetylation/deacetylation, the most extensively studied type of histone modification involved in transcription control, in a mouse model of cardiac fibrosis. They found that the inhibition of histone deacetylase (HDAC) activity targeted to one subclass of HDACs (class I) suppressed cardiac fibrosis. However, the effects of HDACs on cardiac fibrosis can be indirect. They can reflect histone acetylation changes that affect cell signaling or transcription factor (TF) activity rather than directly targeting the gene of interest, and they can involve acetylation of non-histone proteins [4, 13]. In addition to chromatin epigenetic analyses, disease- and transcriptionassociated changes in DNA methylation were found in the promoter regions of fibrosis- and inflammation-related genes $[4,14,15]$.

Among the major contributors to cardiac fibrosis and related heart disease are the deleterious activation of the autocrine/paracrine renin-angiotensin system (RAS), excessive inflammatory signaling from infiltrating monocyte/macrophages, and pathological ECM remodeling upon upregulation and transdifferentiation of cardiac fibroblasts or infiltrating fibrocytes [16-18]. We chose genes from each of these pathways to study relationships between tissue-specific epigenetics and transcription and to identify regions in the vicinity of these genes that are candidate cis-acting elements contributing to gene dysregulation in cardiac fibrosis. These genes are AGT (Angiotensinogen), NLRP3 (NLR Family Pyrin Domain Containing 3), CCN2/CTGF (Cellular Communication Network Factor 2/Connective Tissue Growth Factor), and MMP2 and MMP9 (Matrix Metallopeptidase 3 and 9). AGT encodes the precursors of angiotensin II (Ang II), a fibrosis-inducing growth factor and signaling molecule as well as an endocrine vasoconstrictor [19], which can be expressed by myofibroblasts as well as by liver and other organs [17]. Ang II is on one of the pathways for priming and activating NLRP3 
inflammasome formation [18] as well as being a key stimulus for cardiac fibrosis [19]. Activation of NLRP3 inflammasomes, which involves upregulation of NLRP3 gene expression, results in the production of IL-1 $\beta$ and IL-18, which, in turn, promotes myofibroblast formation, and can also initiate pyroptosis to induce cardiac inflammation. NLRP3 is also critical for inflammation associated with many other diseases (including cancer, colitis, gout, type II diabetes, and neonatal onset multisystem inflammatory disease) as well as its normal protective roles in responding to a wide range of damage signals [20]. NLRP3 protein can also contribute to fibrosis by favoring the epithelial-to-mesenchymal transition in both inflammasome-dependent and independent pathways [21]. In addition, abnormal NLRP3 inflammasome signaling can lead to arrhythmic sarcoplasmic reticulum $\mathrm{Ca}^{+2}$ release in the heart promoting atrial fibrillation [18]. CTGF, the product of the $C C N 2 / C T G F$ gene, is an autocrine regulator of fibrosis [16] with many disparate cell type-specific effects including the induction of angiogenesis [22, 23]. It upregulates cardiac fibrosis through its stimulation of cardiac fibroblasts although it is also upregulated in injured cardiomyocytes [16]. MMP2 and MMP9 are closely related type IV collagenases that help shape the ECM and may provoke or ameliorate cardiac fibrosis depending on the context [5].

Despite the importance of these genes to cardiac fibrosis and the major role of enhancers (chromatin that upregulates, in cis, promoters at the 5' end of genes and is often distant from the promoter) in tissue-specific gene expression [6], analysis of the epigenetics of these genes has been mostly limited to promoter regions [14, 23-27]. However, there have been notable exceptions $[14,25,28,29]$. In the present study we describe important epigenetic features within gene bodies and in the gene neighborhoods of NLRP3, MMP2, MMP9, CCN2/CTGF, and AGT as well as non-coding RNAs whose dysregulation might contribute to abnormal expression of these genes in disease. 


\section{Materials and methods}

\subsection{Transcriptomics}

RNA-seq data for tissues and skin fibroblast cell lines were obtained from the Genotype-Tissue Expression database (GTEx) using median gene expression levels derived from hundreds of samples for each tissue type. [30]. For cell cultures, RNA-seq data were from poly(A) ${ }^{+}$RNA in color-coded overlaid graphs in the figures (ENCODE/Wold Lab at Caltech; vertical viewing range, $0-8$ except for $A G T$ for which it was 0 - 12; UCSC Genome Browser [31]) with quantification of RNA-seq data as previously described [32]. In addition, strand-specific RNAseq profiles (ENCODE/Cold Spring Harbor Labs[31]) were used to confirm which strand was transcribed and to examine additional cell types (UCSC Genome Browser [31]).

\section{2 Epigenetics}

Epigenetic data were from the Roadmap Epigenetics Project [6] downloaded from UCSC Genome Browser hubs (http://genome.ucsc.edu/) [31]. The 25-state chromatin state segmentation tracks were used except for $C C N 2$ for which the 18-state tracks were used because they gave fewer uninformative "low-signal" segments. The original color-coding of Roadmap data for chromatin states as shown in the figures was slightly modified to increase clarity. The displayed histone acetylation profiles (vertical viewing range 0 - 10) were the same ones that had been used in determination of chromatin state segmentation [6]. The tissues for chromatin states were from males except for lung, monocytes, and B cells $[6,31]$ and prefrontal cortex (a mixture of 81-yo male and 75-yo female samples), and cell cultures were previously described [32]. DNaseI hypersensitivity profiling (vertical viewing range 0 - 40) and DNA methylation profiling by bisulfite-seq usually came from the same samples as for the chromatin state [6]. Additional bisulfite-seq profiles for a control and an atherosclerotic aorta sample (Aorta ctl \#2, thoracic 
aorta, and Atheroscl aorta, aortic arch) came from an 88 yo female [33] and are available at the UCSC Genome Browser (93N and 93A, Zaina-2014 at the DNA Methylation Hub). Atherosclerosis-specific and aorta-specific DNA hyper- or hypomethylation was determined from bisulfite-seq data as previously [34]. At the UCSC Genome Browser, the monocytes for the bisulfite-seq track are incorrectly labeled as macrophages. Super-enhancer designations were from the dbSUPER website [35] and checked by inspection of histone acetylation tracks at the UCSC Genome Browser.

\subsection{Transcription factor binding profiles}

A limited number of whole-genome $\mathrm{TF}$ binding profiles obtained by chromatin immunoprecipitation/next-gen sequencing (ChIP-seq) on cell types expressing the genes highlighted in this study are publicly available. The ENCODE 3 Transcription Factor ChIP-seq Peaks provided the examined TF binding data (UCSC Genome Browser [31].

\section{Results}

3.1 The inflammasome-associated NLRP3 gene has a super-enhancer mirroring its high and specific expression in monocytes and neutrophils

NLRP3 encodes a protein that is a limiting factor for activation of NLRP3 inflammasome formation [36], which is important in inflammation-related cardiovascular disease [37]. It is expressed at a much higher level in leukocytes (median of 755 samples, 23 TPM (transcripts per kilobase million; GTEx RNA-seq database [30]) than in any of the 49 other examined normal tissues (Fig. 1A). NLRP3 displayed enhancer chromatin (orange segments) within the gene body and surrounding the gene specifically in monocytes, neutrophils, and lymphocytes (Fig. 2B); macrophage epigenetic data are not available. Enhancer chromatin had been determined from 
enrichment in histone H3 lysine 27 acetylation (H3K27ac) plus H3 lysine 4 monomethylation (H3K4me1) [6]. Active promoter chromatin (Fig. 2B, red segments) at the transcription start site

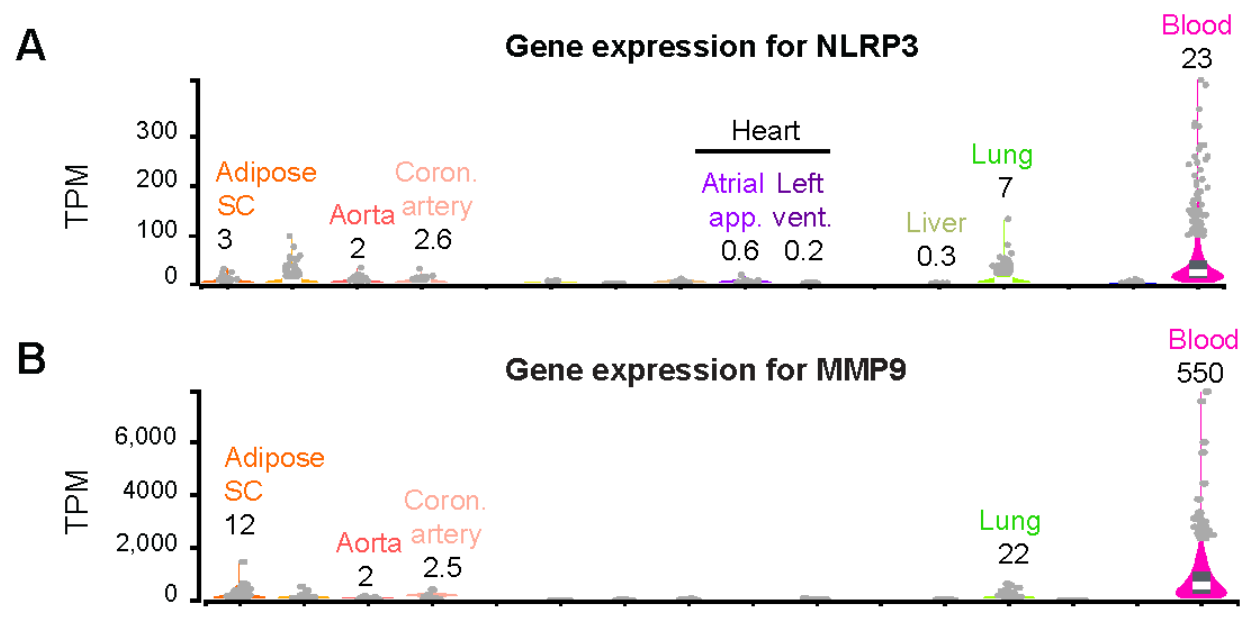

C

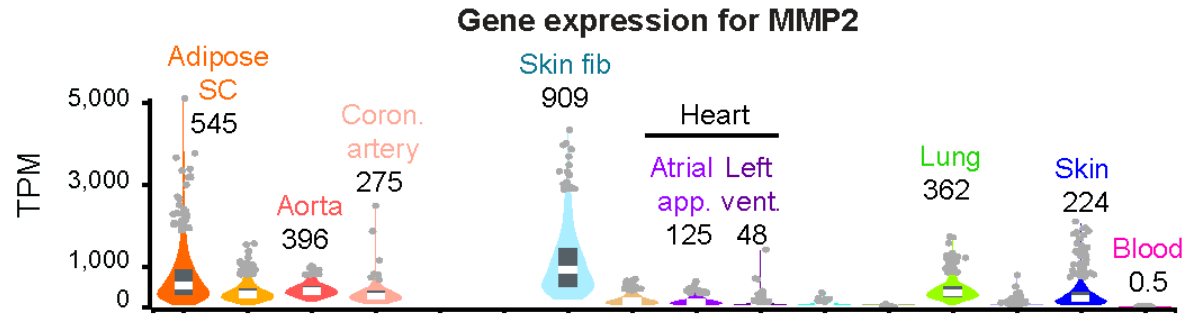

D

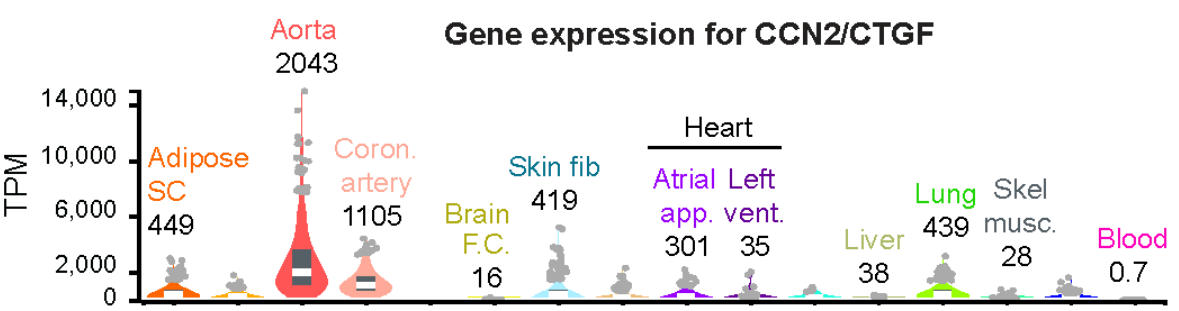

E

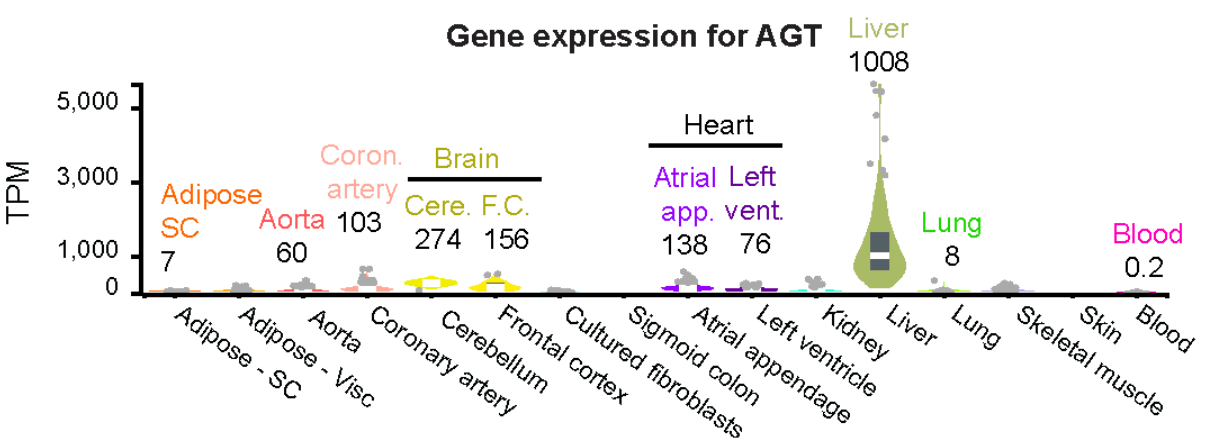

Fig. 1. Tissue-specificity of gene expression is similar for $N L R P 3$ and $M M P 9$ but dissimilar for $M M P 2, C C N 2 / C T G F$, and $A G T$. RNA-seq data (violin plot) for expression of the indicated genes in 15 tissues and in cultured skin fibroblasts is shown; there were hundreds of biological replicates for each tissue type. Select median values are given. The tissues shown are a subset of the 52 
tissues in the GTEx database [30]. Adipose SC, subcutaneous adipose; Coron., coronary; brain FC, brain frontal cortex; cere., cerebellum; atrial app., atrial appendage; vent, ventricle; blood, leukocytes.

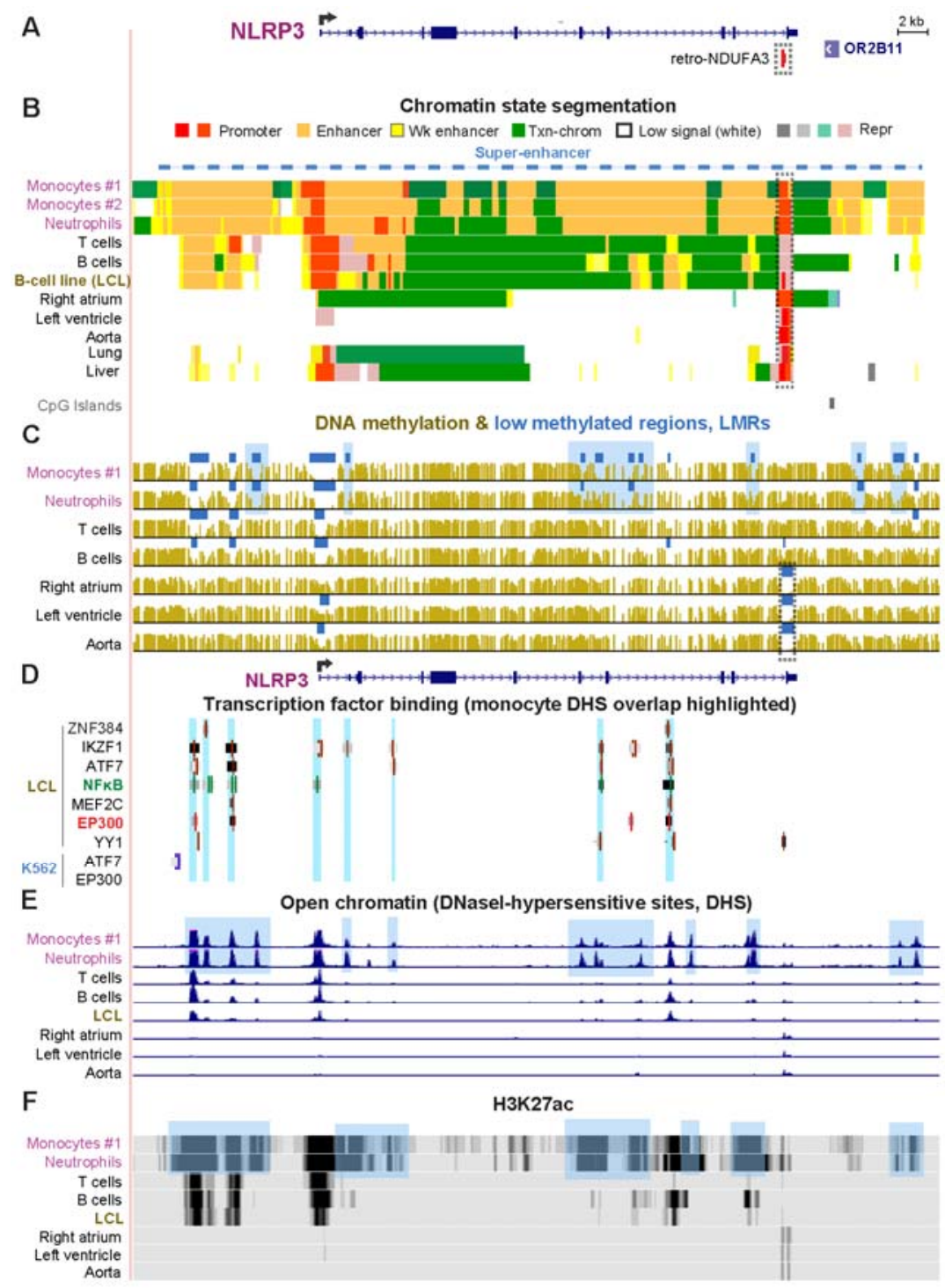

Fig. 2. NLRP3 exhibits a super-enhancer containing foci of DNA hypomethylation and DNaseI hypersensitivity specifically in monocytes and neutrophils, which highly express this gene. (A) The main NLRP3 (chr1:247,566,540-247,622,212) RefSeq isoform is shown (see Supplemental Fig. S1) and the adjacent olfactory gene OR2B11, which is silent in all studied tissues. Broken arrow, the TSS and direction of transcription. (B) Chromatin state segmentation denotes predicted promoter (red), strong enhancer (orange), weak enhancer (yellow), low-signal or repressed (Rep), or H3K36me3-enriched transcribed (green) types of chromatin. Two monocyte samples are shown for the chromatin state analysis. Blue dashed line, super-enhancer in monocytes and neutrophils. (C) DNA methylation profiles (bisulfite-seq); blue bars, regions that were significantly hypomethylated relative to the same genome (LMRs). (D) Local binding of the TFs in the lymphoblastoid cell line (LCL) or in K562 cells. (E) Open chromatin peaks determined as DNaseI-hypersensitive sites (DHS). (F) H3K27ac signal intensity, indicative of the strength of the promoter and enhancer chromatin. Blue highlighting in (C), (E), and (F), monocyte/macrophage- 
specific epigenetic marks. All tracks are from hg19 in the UCSC Genome Browser and are aligned in this and subsequent figures.

(TSS; Fig. 2A, broken arrow) was seen in these leukocytes but also in liver, which had very little NLRP3 RNA and lacked appreciable amounts of enhancer chromatin. Active promoter chromatin had been determined from enrichment in H3K27ac and H3K4me3, instead of the H3K3me1 enrichment that helps define enhancers [6]. Importantly, NLRP3 did not just display traditional enhancers in monocytes and neutrophils, but rather a super-enhancer, which is an unusually large cluster $(>3 \mathrm{~kb}$ ) of enhancer chromatin regions that can include some promoter chromatin (Fig. 2B, dotted line) $[35,38]$. Super-enhancers are typically associated with especially strong, differentiation-related expression of the related gene.

Within the NLRP3 super-enhancer, there were regions of especially low DNA methylation (low methylated regions, LMRs; blue horizontal bars, Fig. 2C) that were specific to monocytes and/or neutrophils. The monocyte/neutrophil-associated LMRs usually overlapped myeloid-specific regions of open chromatin (DNaseI-hypersensitive sites, DHS) and of high enrichment in H3K27ac (Fig. 2C - E, blue highlighting). A subregion containing 3 CpGs located $0.6 \mathrm{~kb}$ upstream of the TSS that was previously shown to be hypomethylated in a monocytic leukemia cell line upon infection with a myobacterium [14] was less methylated in monocytes than in other samples (Supplemental Fig. S1G, blue highlighting).

$\mathrm{B}$ cells and T cells, like monocytes and neutrophils, were enriched in promoter chromatin (Fig. 2B) at the NLRP3 TSS but had much less enhancer chromatin in and around NLRP3. Bcells and T-cells were reported to have negligible NLRP3 expression unless expression was induced, e.g., by lipopolysaccharide [39, 40]. RNA-seq profiles indicated a low level of 
expression of NLRP3 in resting B cells and in a B-cell lymphoblastoid cell line (LCL; GM12878) (Supplemental Fig. S1B). Genome-wide profiles of binding by many TFs were available for this LCL and for K562 cells (myelogenous leukemia cell line) [31, 41]. TFs bound in or near open chromatin regions (DHS) in NLRP3 in the LCL, but not in the analogous regions in NLRP3-nonexpressing K562 cells (Fig. 2D and E). These TFs include ZNF384, a regulator of extracellular matrix proteins; IKZF1 (IKAROS), a regulator of hematopoietic cell differentiation; ATF7 and MEF2C, transcriptional activators, EP300, a histone transacetlylase, chromatin remodeling protein, and enhancer/super-enhancer protein; and the RELB subunit of NFkB (Fig. $2 \mathrm{D}$, green lines). NFkB is a signaling TF that is necessary, but not sufficient, for transcriptional activation of NLRP3 [36]. Many more DHS probably bind EP300 in monocytes and neutrophils than in the weakly NLRP3-expressing lymphoblastoid cells; these TF-binding profiles were not available for monocytes or neutrophils.

Surprisingly, we found that the distal (3') end of NLRP3 displayed promoter chromatin with the histone marks of active (Fig. 2B, red segments) or poised/repressed (beige segments) promoters in most tissues. This 3' promoter chromatin (Fig. 2B, dotted rectangle) overlapped a retrotransposon-derived gene, retro-NDUFA3 near the end of the last intron (Fig. 2A). Only leukocytes had high levels of DNA methylation in this region (Fig. 2C and 10 additional tissue types not shown). These findings suggest that leukocyte-specific DNA methylation repressed most, but not all (Supplemental Fig. S1D), of the 3' promoter activity that would interfere with NLRP3 mRNA formation in monocytes and neutrophils. Given the enhancer chromatin adjacent to the 3' promoter chromatin in monocytes and neutrophils (Fig. 2B), it may be especially important to have local DNA methylation in these cell types. ChIP-seq indicated that the TF YY1, a transcriptional repressor or activator protein, binds within this promoter region (Fig. 2D). Similarly, we had previously demonstrated for CDH15 and PITX3 that DNA hypermethylation 
specific to tissues expressing these two genes repressed an intragenic 3' promoter [42]. Downstream of NLRP3 is a gene encoding an olfactory receptor protein (OR2B11) that contains regions of enhancer chromatin in monocytes and neutrophiles despite its negligible expression in all 52 tissues from the GTEx database. Therefore, the enhancer chromatin in the vicinity of $O R 2 B 11$ probably upregulates expression of $N L R P 3$.

We also examined genes encoding the other three components of NLRP3 inflammasomes. CASP1 and PYCARD (ASC) displayed DNA hypomethylation and large amounts of enhancer chromatin specifically in monocytes and neutrophils (CASPI) or in leukocytes in general (PYCARD; Fig. S2A and B). In contrast, NEK7 showed less tissue specificity in its expression and epigenetics and had a lower level of RNA in leukocytes than in most other tissues (Fig. S2C). Vento-Tormo et al. [15] examined monocyte-macrophage differentiation-related changes immediately upstream of the promoter regions of PYCARD and CASP1 and found that the differentiation of monocytes was accompanied by DNA hypomethylation in these regions concomitant with gene upregulation. In the methylated subregions that we saw immediately upstream of the promoter, we noted enhancer chromatin in monocytes (Fig. S2A and B). The inferred enhancer might increase in strength upon demethylation during differentiation to macrophages.

\subsection{The gene encoding miR-223, which downregulates NLRP3 protein production, has a} monocyte- and neutrophil-specific super-enhancer, like NLRP3

MicroRNA (miRNA) miR-223, is a critical regulator of NLRP3 protein levels by decreasing NLRP3 mRNA stability and translation [43]. Its host gene, hsa-mir-223, is transcribed preferentially in leukocytes as well as in spleen (TPM, 185 and 146, respectively) unlike NLRP3 which is transcribed preferentially only in leukocytes (TPM, 23 and 6, respectively). We found that the 5-kb hsa-mir-223 gene is embedded in a $38-\mathrm{kb}$ super-enhancer 
seen specifically in monocytes and neutrophils (Fig. 3D, dashed line). The super-enhancer overlapped DHS and pockets of DNA hypomethylation that were most prominent in monocytes and neutrophils (Fig. 3E and G, blue highlighting) and stopped at the end of the neighboring VSIG4 gene, which has a different tissue specificity. 
bioRxiv preprint doi: https://doi.org/10.1101/2020.10.23.352518; this version posted October 24, 2020. The copyright holder for this preprint (which was not certified by peer review) is the author/funder, who has granted bioRxiv a license to display the preprint in perpetuity. It is made available under aCC-BY 4.0 International license.

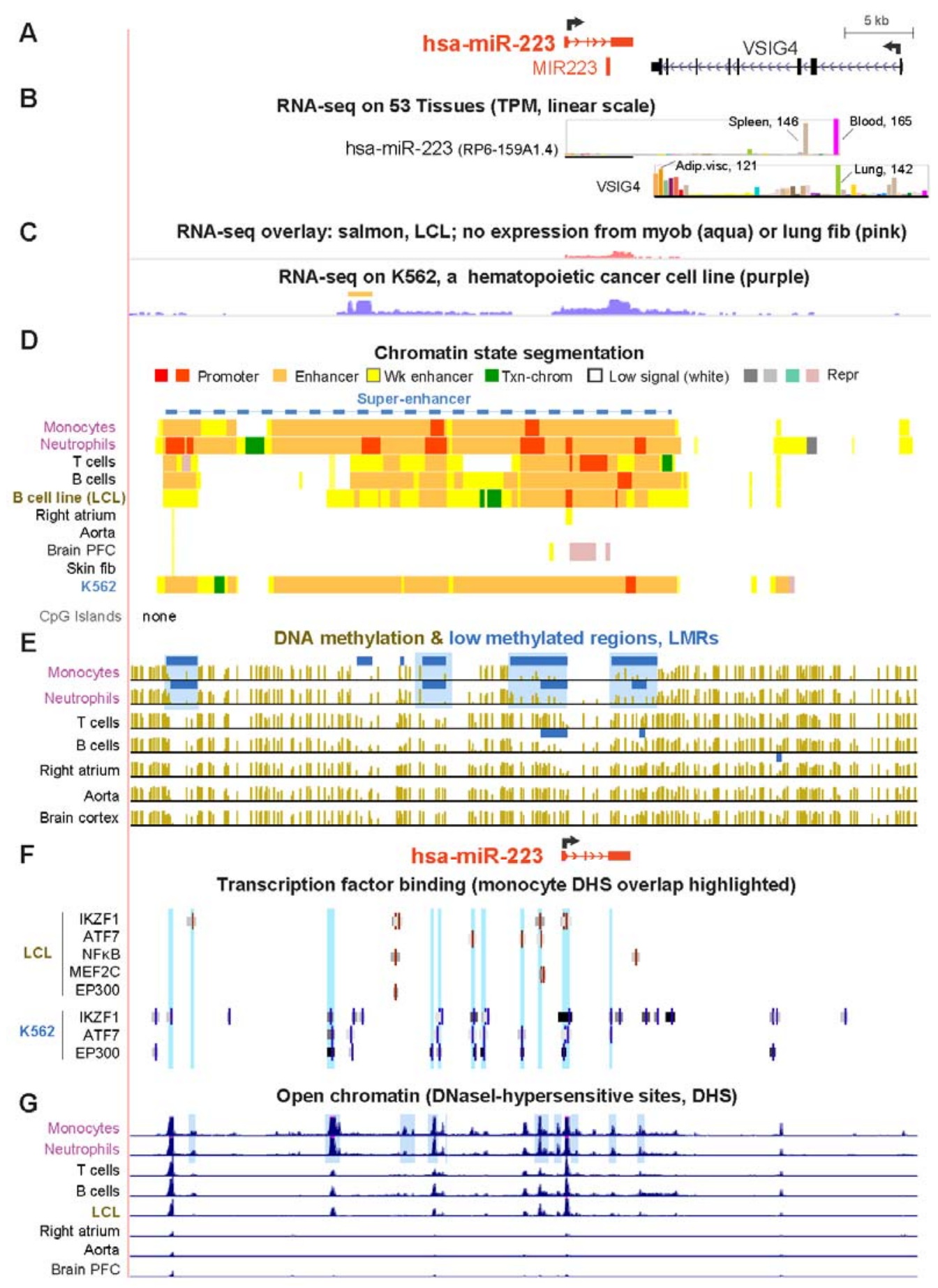

Fig. 3. The host gene encoding the microRNA miR-223 has a super-enhancer over the gene that extends far upstream. (A) $h s a-m i R-223$ and its gene neighborhood (chrX:65,203,177-65,260,919). (B) Tissue expression (bar graphs) from GTEx with selected mean TPM indicated. The thick horizontal line shows the region used for TPM determination. (C) RNA-seq for cell cultures is given for the first track as overlaid color-coded signal from myoblasts and lung fibroblasts and, for the second track, the data are just for the K562 cells. (D), (E), (F) and (G) and highlighting are as in Fig. 2. Blue dashed line, super-enhancer in monocytes and neutrophils; PFC, prefrontal cortex of brain. A second sample of monocytes from males (not shown) gave a similar chromatin state segmentation profile as for the exhibited female sample. 
Less extensive, traditional enhancer chromatin was observed in T cells, B cells, and a Bcell derived LCL (GM12878; Fig. 3D and F, blue highlighting). The LCL as well as the hematopoietic cancer-derived K562 cell line preferentially express the miR-223 miRNA precursor with especially high levels in K562 cells, which also display a super-enhancer spanning the gene (Fig. 3C). A novel long noncoding RNA (lncRNA; Fig. 3C, orange line) as well as hsa-mir-223 is strongly expressed from the K562 super-enhancer. This lncRNA is likely to be associated with the K562 enhancer activity but is stable, unidirectional (plus-strand RNAseq, data not shown) and produced in considerable amounts unlike the typical enhancerassociated RNA (eRNA), which is short, bidirectional, and difficult to detect due to instability [44]. The LCL displayed none of this lncRNA, had much less extensive enhancer chromatin, and had much less TF binding in the distal region than did K562 cells (Fig. 3F).

\subsection{Fibrosis-associated MMP9 and MMP2 genes display very different tissue-specific expression} and epigenetic profiles

$M M P 2$ and $M M P 9$, paralogous genes that encode related matrix metalloproteinases and are critically involved in cardiac fibrosis and cardiovascular disease [5], have very different tissue specificities. The concentrations of their respective processed protein products must be carefully balanced with levels of tissue inhibitors of metalloproteinases (TIMPs). MMP9 is expressed preferentially in leukocytes (Fig. 1B). In contrast, MMP2 is highly expressed in a broad spectrum of tissues, e.g., skin, aorta, lung, and adipose, and in many progenitor cell types, including skin and lung fibroblasts (Fig. 1C, 4; Supplemental Table S1). Curiously, MMP2 as well as $A G T$ and $C C N 2 / C T G F$ displayed much higher expression in the right atrial appendage than in the left ventricle (Fig. 1), the only two regions of heart with expression data in the GTEx database. From the multiple MMP2 isoforms containing different TSSs, 5' Cap Analysis of Gene Expression (CAGE [31]) indicated that one predominant TSS is used in the examined cell 
bioRxiv preprint doi: https://doi.org/10.1101/2020.10.23.352518; this version posted October 24,2020 . The copyright holder for this preprint (which was not certified by peer review) is the author/funder, who has granted bioRxiv a license to display the preprint in perpetuity. It is made available under aCC-BY 4.0 International license.

cultures (Fig. 4B, broken arrow). Curiously, the 3' end of MMP9 overlaps the 3' end of SLC12A5-AS1, a gene encoding a lncRNA whose 5' end overlaps a brain-specific gene SLC12A5. However, this lncRNA gene, which has overall low expression, has an expression profile that is a mixture of that of $M M P 9$ and SLC12A5 but more like that of MMP9 [30]. Therefore, this lncRNA might help regulate both protein-coding genes that it overlaps.
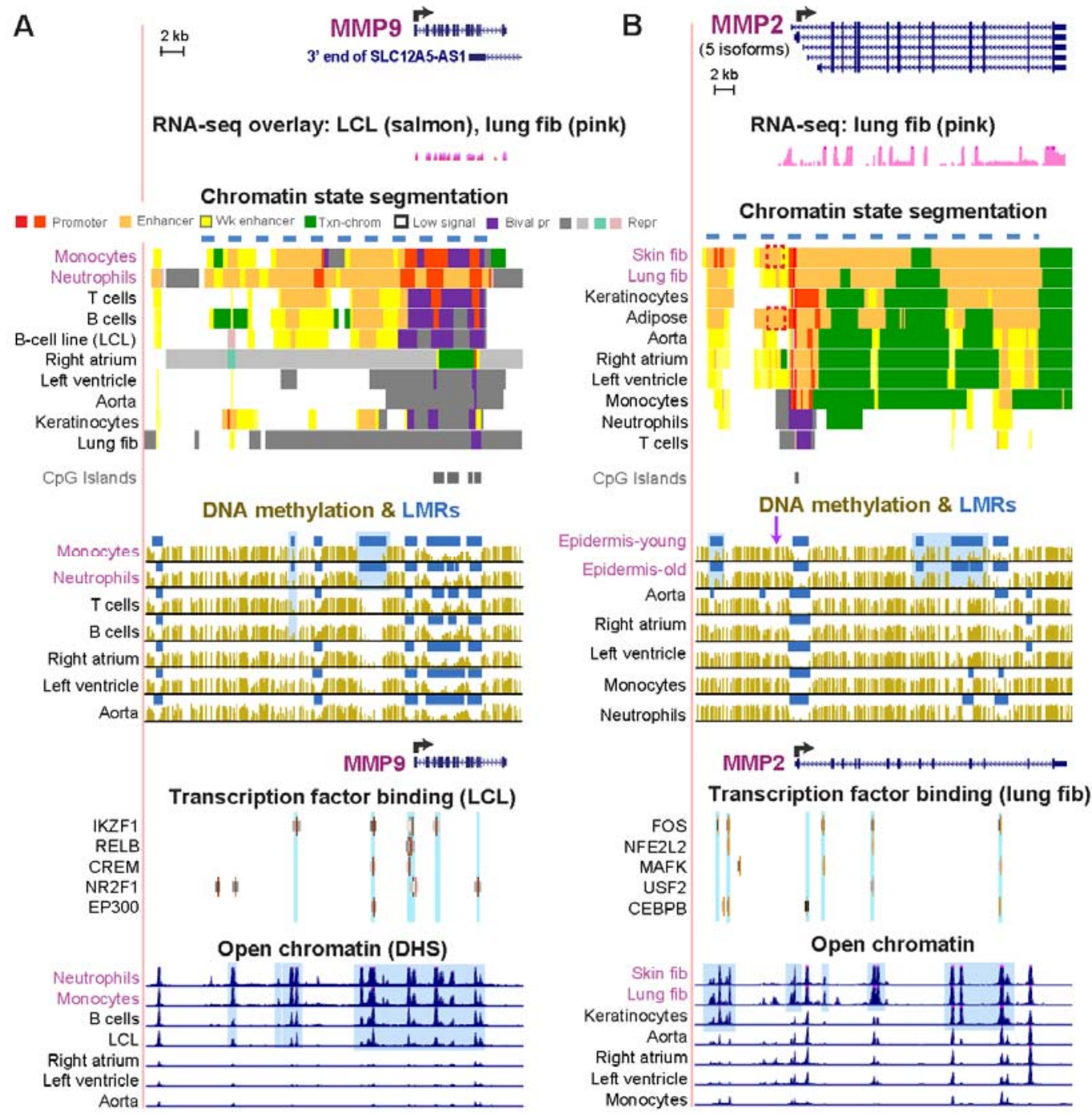

Fig. 4. $M M P 9$ has a super-enhancer in neutrophils while $M M P 2$ has one in skin and lung fibroblasts. (A) $M M P 9$ (chr20:44,615,045-44,646,541) and (B) $M M P 2$ (chr16:55,503,06355,541,196) as in Figs. 2 and 3. Blue highlighting, LMRs or DHS that are specific for monocytes/neutrophils or leukocytes; light blue highlighting for TF binding, TFs binding to DHS that are specific for leukocytes or fibroblasts/keratinocytes. Fib, fibroblast; bival pr, bivalent (repressed or weak) promoter chromatin containing H3K4me3 and H3K27me3. 
We found super-enhancers in neutrophils and skin fibroblasts/lung fibroblasts, for MMP9 and $M M P 2$, respectively (Fig. 4A and B, dashed lines over the chromatin state tracks), which are likely to drive the high expression of these genes in these cell types. MMP9 displayed more DNA hypomethylation and stronger DHS in monocytes and neutrophils than in B cells or the Bcell LCL (Fig. 4A, blue highlighting) consistent with cell type-specific differences in the extent of enhancer chromatin. Available TF-binding profiles for MMP9 in the LCL and MMP2 in a lung fibroblast cell strain showed that most of the TF binding was again in expression-associated DHS (Fig. 4, light blue highlighting). In the case of $M M P 2$, tissue-specific DNA hypomethylation was most prominent in skin epidermis (for which no chromatin state profile is available). This could be related to the high level of $M M P 2$ expression in skin tissue (epidermal plus dermal layers; Fig. 1C). Although skin and aorta both exhibit very high levels of $M M P 2$ expression, skin displayed much more DNA hypomethylation than aorta, suggesting that different epigenetic strategies are sometimes used for maintaining similar levels of tissuespecific expression of a given gene (Figs. 1C and 4B). Heart failure-linked DNA hypomethylation was previously found [25] near $M M P 2$ and $C C N 2$ (Figures 4B and 5B, purple arrows), as discussed below.

\subsection{The liver-specific expression of AGT matches tissue-specific profiles of promoter and} enhancer chromatin, open chromatin, and DNA methylation

$A G T$, a gene with critical functions in regulating blood pressure and vascular remodeling, is expressed highest in liver (Fig. 1E). Accordingly, liver and HepG2 cells displayed the most intragenic and intergenic promoter and enhancer chromatin and DNA hypomethylation as well as much TF binding (Fig. 5A, dotted boxes). Brain and heart, which had high levels of AGT RNA, 
bioRxiv preprint doi: https://doi.org/10.1101/2020.10.23.352518; this version posted October 24, 2020. The copyright holder for this preprint (which was not certified by peer review) is the author/funder, who has granted bioRxiv a license to display the preprint in perpetuity. It is made available under aCC-BY 4.0 International license.

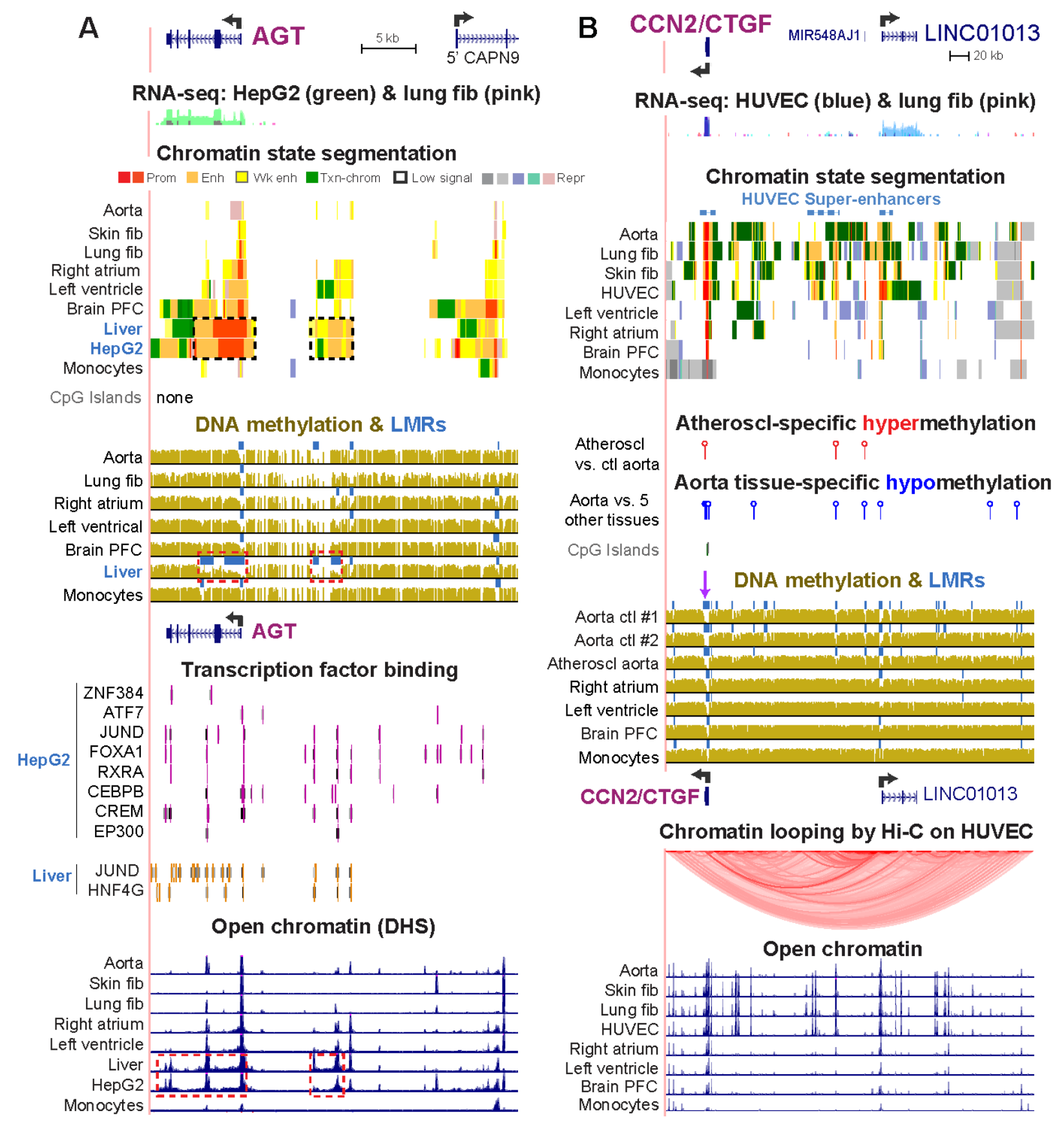

Fig. 5. Enhancers in neighboring genes that are associated with expression in $A G T$ or $C C N 2 / C T G F$. (A) AGT (chr1:230,835,715-230,892,908) and (B) CCN2/CTGF (chr6:132228520132613558) as in Figs. 2 - 4 with the following additions. Significant differentially methylated regions (DMRs) in aorta vs. five other tissues (heart, skeletal muscle, lung, adipose or monocytes) and in an atherosclerotic (Atheroscl) aorta sample vs. three control (Ctl) aorta samples were determined for $C C N 2$ from bisulfite-seq data; blue, hypomethylated DMR; red, hypermethylated DMR. In addition, a chromatin looping profile [31, 45] (Hi-C technique) is shown for umbilical vein endothelial cells (HUVEC). 
although not as high as liver (Fig. 1E), exhibited considerable intragenic and intergenic AGT enhancer chromatin but less than for liver (Fig. 5A). In brain, liver, and HepG2, there was additional enhancer and promoter chromatin overlapping the gene body of $A G T$ 's nearest upstream neighbor CAPN9, which encodes a cysteine protease. The enhancer profile at CAPN9 paralleled the strong expression of $A G T$ in these tissues (Fig. 1) but was at odds with the negligible expression of CAPN9 [30] in these samples and did not correlate with expression of any other nearby gene. Therefore, we propose that this enhancer chromatin at CAPN9 in liver and brain is upregulating its neighbor, $A G T$, rather than itself.

Previous studies revealed enhancer activity or enhancer chromatin only in part of the body of the AGT gene. Both an 80-bp associated enhancer element in the last exon of $A G T$ that was active in HepG2 cells and a 1.4-kb androgen-responsive enhancer in intron 2 of $A G T$ were described [28, 29]. The involvement of even more enhancer chromatin in regulating expression of $A G T$ in liver and HepG2 cells is indicated not only by our description of their epigenomic profiles above, but also, by the many positively-acting TF binding sites throughout the gene and far upstream (Fig. 5A).

3.5 Preferential expression of CCN2/CTGF in aorta is accompanied by far-upstream DNA hypomethylation and enhancer chromatin over a cancer-associated ncRNA gene (LINC01013)

$C C N 2 / C T G F$, which is involved in many aspects of development (including angiogenesis) and homeostasis as well as in diseases (e.g., myocardial infarction, atherosclerosis, hypertension, and cancer $[22,23])$ had a very high median level of RNA (TPM, 2043) in aorta. Most examined cell cultures, including skin fibroblasts, lung fibroblasts, chondrocytes, umbilical vein endothelial cells (HUVEC) and HepG2 cells highly express this gene and displayed multiple regions of enhancer chromatin within a 350-kb region upstream of CCN2 that includes 
several ncRNA genes (Figs. 1D and 5B; Supplemental Figs. S3 and S4). Many TFs colocalized with cell type-specific DHS in this region. HUVEC, which had the highest expression of CCN2 among examined cell cultures (Supplemental Table S1), displayed the highest expression of LINC01013, a little-studied ncRNA gene in its far upstream intergenic region (Fig. 5B and Supplemental Fig. S4E). Coordinate expression of LINC01013 and CCN2 was previously observed in HUVEC after stimulation of the angiogenesis program genes in vitro with VEGFA [46]. The only other cell type expressing this ncRNA was chondrocytes. Little else is known about LINC01013 except that it can repress chondrogenesis [47], and is associated with cancer progression [48]. Profiles of higher-order looping domains (topologically associating domains, TADs) in this neighborhood gave evidence for a loop between $C C N 2$ and the immediate upstream region of LINC01013 in HUVEC (Fig. 5B, Hi-C [45]), but not in an LCL or a keratinocyte cell line (data not shown), which have negligible expression of LINC01013 (Supplemental Table S1). Therefore, the evidence supports the hypothesis that transcription of LINC01013 upregulates CCN2 in HUVEC. However, coordinate expression between CCN2 and LINC01013 was seen only in HUVEC and chondrocytes and not in any tissue, including aorta. Therefore, any linkage of expression of these two genes is restricted to only some CCN2expressing cell types.

The very high expression of $C C N 2$ in aorta was accompanied by less enhancer chromatin and weaker DHS in this region than seen in HUVEC or lung fibroblasts. HUVEC displayed three super-enhancers in this gene neighborhood, over CCN2, LINC01013, and an intergenic region in between them (Fig. 5B, dotted blue lines), but no super-enhancer was found in aorta. In interpreting the relationship between expression of this gene and the enhancer chromatin based upon GTEx RNA-seq data, we considered the possibility that CCN2/CTGF expression in GTEx samples was biased upward because $\sim 38 \%$ of the hundreds of GTEx aorta samples were from 
individuals of $>60$ yo. In contrast, the donor for the aorta chromatin state and DNA methylation analysis and for many other Roadmap samples [6] was a 34-yo male. However, this was not a problematic comparison because when we stratified GTEx data for expression of CCN2/CTGF aorta by age, the median values for expression of $C C N 2 / C T G F$ for 59 donors between 20 and 49 yo were not significantly different from that for 42 donors between 50 and 79 yo.

One of the biggest differences between the epigenetics of the CCN2 gene neighborhood in aorta and in other less highly expressing tissues was the presence of aorta-associated DNA hypomethylation. There were many aorta-specific LMRs at CCN2 or far upstream of the gene that showed statistically significant DNA hypomethylation in aorta vs. the set of heart, lung, skeletal muscle, and monocyte samples (Fig. 5B, blue lollipops). Several of these LMRs overlapped aorta/HUVEC/fibroblast-associated DHS. Bisulfite-seq DNA methylation profiles were available for two samples from an 88-yo female [33] and one from the 34-yo Roadmap control aorta (Fig. 5B, Aorta ctl \#1 [6]). One of the 88 yo donor's samples was normal-appearing thoracic aorta (Aorta ctl \#2) and the other from the same donor was a section of atherosclerotic aortic arch (Atheroscl aorta). Similar distinctive patterns of DNA hypomethylation were seen in all examined aorta samples (Fig. 5B, blue lollipops) but there was a small but significant amount of DNA hypermethylation in the atherosclerotic aorta vs. the control aorta samples in the vicinity of CCN2 or LINC01013 (Fig. 5B, red lollipops). Similarly, we and Zaina et al. [33, 34] previously showed that throughout the genome there were many more hypermethylated than hypomethylated DNA regions in atherosclerotic vs. control aortas. It remains to be determined if there are decreases in enhancer or promoter chromatin in some atherosclerosis-associated hypermethylated DNA regions.

\section{Discussion}


The fibrosis-related protein-coding genes that we studied, $N L R P 3, M M P 2, M M P 9$, $C C N 2 / C T G F$, and $A G T$, are upregulated in diverse diseases. These include cardiac fibrosis and atherosclerosis [18, 19, 23], liver and lung fibrosis [23, 26, 49], rheumatoid arthritis and osteoarthritis [26], many types of cancer [23, 24, 50], and age-related increased susceptibility to vascular disease [51] and to lethality from COVID-19 [2]. The precise control of transcription of these genes is important because they can participate in restorative as well as pathological repair [52] or inflammation [18]. Age-related disease susceptibility to fibrosis and fibrosis-associated cardiovascular disease can be driven, in part, by age-related build-up of epigenetic mistakes in key genes [8]. Some of these mistakes might help abnormally upregulate expression of the studied genes, which are usually overexpressed rather than downregulated in disease. Despite the central role of epigenetics and, in particular, enhancers to gene regulation [38, 44], previous studies of the epigenetics of the genes that were highlighted in this study have been limited.

By using diverse whole-genome epigenetic profiles rather than just data from locusspecific studies [6], we were able to extend previous descriptions [25, 28, 29, 53] of transcription-regulatory chromatin at $A G T, C C N 2 / C T G F$, and $M M P 2$ and describe for the first time enhancer chromatin for NLRP3. NLRP3 protein is crucial for innate immunity and inflammation and is a major player in the induction of fibrosis [18]. Our results indicate that the examined cardiac fibrosis-associated genes, NLRP3, MMP2, MMP9, CCN2/CTGF, and the $N L R P 3$ mRNA-regulatory $h s a-m i R-223$, have previously undescribed super-enhancers (clusters of traditional enhancers) containing foci of DNA hypomethylation, open chromatin, and TF binding that are very likely to help drive the high tissue-specific expression of these genes. Super-enhancers at other genes have been implicated in cardiovascular disease $[11,54] . A G T$, the other fibrosis-related gene that we studied, has traditional enhancer chromatin as well as elongated promoter chromatin that can account for its very high expression in liver. Although 
enhancers are often thought of as upstream or downstream of genes, all six genes contained some of their enhancer chromatin in the gene body. Such gene-body enhancer regions might facilitate rapid transcription elongation as well as potentiate promoters, their better known role [55].

A caveat in our study is that our comparison of epigenetic and transcription profiles relies on steady-state mRNA levels, which depend, in part, upon factors affecting the stability of the RNA and, thereby, the measured mRNA levels. However, miRNA-targeted degradation usually only fine-tunes target mRNA levels, typically about two-fold [56]. Nonetheless, such fine-tuning can have important biological consequences, and more than one miRNA can target an mRNA. miR-223, which is encoded by the $h s a-m i R-223$ gene (Fig. 3), targets NLRP3 mRNA as well as mRNAs for inflammation-related signaling proteins and TFs, downregulates healing of slowhealing wounds, upregulates granulopoiesis, downmodulates monocytic/macrophage differentiation, regulates the innate immune response in chronic obstructive pulmonary disease and asthma, and can affect cell viability, proliferation, and invasion in carcinogenesis [57-59].

The microRNA miR-223 as well as TTP, an RNA-binding protein that reduces levels of NLRP3 mRNA, specifically target a long-form polyadenylation variant and not a short-form variant with a shorter 3' untranslated region [60]. Importantly, there are similar amounts of both NLRP3 RNA isoforms in macrophages, which we did not study because of a lack of available epigenome profiles, but the short-form variant is the predominant one in monocytes [60]. As monocytes differentiate to macrophages, there are decreases in miR-223 and increases in NLRP3 protein due to increased translation despite decreases in steady-state levels of NLRP3 mRNA [43]. We found that $N L R P 3$ mRNA and the pri-miRNA precursor of miR-223 were most highly expressed in leukocytes, and the genes for both had super-enhancers specifically in monocytes and neutrophils (Figs. 1 - 3). The upregulation of NLRP3 transcription as well as the downregulation of the activity of many NLRP3-targeting miRNAs, including miR-223, drive 
inflammasome activation in neutrophils and monocytes/macrophages in response to a priming trigger [27, 36, 43, 59]. Surprisingly, miR-223 is much more highly expressed in leukocytes than is NLRP3. These findings underscore the importance to NLRP3 inflammasome formation of posttranscriptional downregulation of miR-223 activity, probably by sponge or competing endogenous RNAs such as one that has been described to inhibit another mRNA target of miR223 [61]. An additional important regulator of NLRP3 inflammasome formation involves direct modification of the NLRP3 polypeptide by ubiquitylation, sumoylation, and phosphorylation to control NLRP3 protein levels and activity [62]. The multiplicity of controls on this key inflammasome-forming protein indicates how important its precise levels are for its roles in innate immunity, inflammation, and disease $[18,62]$.

Our findings further elucidate previously reported disease-related epigenetic changes in several of the studied genes. A subregion $1.4 \mathrm{~kb}$ upstream of the distal TSS of MMP2 was shown by Glezeva et al. [25] to be hypomethylated in left ventricle samples from heart-failure patients relative to analogous control samples. A disease-linked increase (almost 3-fold) in expression of MMP2 accompanied this hypomethylation. Glezeva et al. had notated this $0.5-\mathrm{kb}$ region as one without regulatory function. We found that this region (Fig. 4B, purple arrow) overlapped strong enhancer chromatin in skin fibroblasts and adipose tissue, both of which highly express this gene (Fig. 4B, dotted boxes). Therefore, our analysis of tissue-specific epigenetics in this region supports the importance of their heart-disease related findings about MMP2. Glezeva et al. [25] also found that heart failure was associated with significant DNA hypomethylation in a 1-kb subregion $0.3 \mathrm{~kb}$ downstream of the 3' end of CCN2/CTGF and 3-fold upregulation of this gene's expression. We found that this DNA region is highly methylated and does not overlap enhancer or promoter chromatin in heart samples. In contrast, it is mostly unmethylated and overlaps promoter or enhancer chromatin and expression-linked peaks of open chromatin in lung 
and skin fibroblasts and aorta (Supplemental Fig. S3, purple arrow). Therefore, the diseaseassociated hypomethylation at $C C N 2$ in heart failure described by Glezava et al. [25] might signify disease-related enhancer formation downstream of CCN2.

Despite only limited amounts of genome-wide data being available for TF binding, we found that some important TFs or cofactors bound to transcription regulatory elements in cells expressing these genes. For example, $C T G F$, whose promoter binds the histone acetyltransferase coactivator EP300 (p300) in response to TGF $\beta$ signaling in mouse fibroblasts [63], also binds this protein at far upstream and downstream enhancer chromatin within open chromatin in HepG2 cells (Supplemental Fig. S3). Therefore, the effects of TGF $\beta$ signaling on CTGF expression probably involve enhancer as well as promoter upregulation mediated by EP300. Similarly, many studies show that NFאB is important for induction of transcription of NLRP3 in response to pathogen-associated molecular patterns but reports of NFKB binding to the $N L R P 3$ gene region have described only the $N L R P 3$ promoter and immediate upstream region [36, 49]. We found multiple NFkB binding sites in regions of open chromatin in the body of the NLRP3 gene, far upstream, as well as in the promoter region in a B-cell LCL (Fig. 2D and E), which expresses this gene only weakly. Importantly, these sites displayed much stronger peaks of open chromatin in NLRP3-highly expressing monocytes and neutrophils. Therefore, it is very likely that there are yet more and stronger TF binding sites in these myeloid cells. The examined liver samples in this study had barely detectable expression of NLRP3 RNA, no NLRP3-associated enhancer chromatin, and only some promoter chromatin at the TSS (Fig. 2). However, NLRP3 expression can be induced in liver upon inflammatory stimulation [49]. We predict that this induction will involve the acquisition of monocyte/macrophage-like intragenic and intergenic enhancer chromatin. A comparison of NLRP3 enhancer/promoter chromatin vs. expression levels 
in various tissues (Figs. 1 and 2) showed that amounts of enhancer chromatin were a better predictor of expression than were amounts of promoter chromatin.

Our study shows the importance of extending epigenetic studies of pathogenic samples to include intragenic and far distal enhancers as well as super-enhancers. By examining the epigenetics of whole gene neighborhoods, we obtained novel insights into regulation of these genes during normal development. For example, our study implicates enhancer chromatin overlapping CAPN9 in liver and brain in the upregulation of AGT, CAPN9's downstream neighbor. CAPN9 itself has undetectable expression in these tissues. In addition, $C C N 2 / C T G F$ and its cancer-related neighbor ncRNA gene LINC01013 [48], which is $182 \mathrm{~kb}$ upstream, provide another likely example of long-distance transcription-regulatory interactions spanning genes but only in HUVEC and chondrocytes. It was previously shown that this gene/lincRNA gene pair exhibit parallel changes in expression after exposure of HUVEC to VEGFA [46]. These types of long-distance interactions in cis, our finding of super-enhancers at most of the studied genes, and the dense clusters of TF binding sites that we describe at these superenhancers provide new insights that can empower the development of drugs aimed at modulating abnormal expression of these important fibrosis-related genes.

\section{Funding}

Financial support for SC came from the Cardiac Arrythmia Fund, a gift made possible through the Headington Foundation. The authors thankfully acknowledge the support in part by U54 GM104940 from the National Institute of General Medical Sciences of the National Institutes of 
Health, which funds the Louisiana Clinical and Translational Science Center (to SC) and from the Tulane Cancer Center.

\section{Acknowledgements}

This research was supported in part using high performance computing (HPC) services provided by Information Technology at Tulane University, New Orleans, LA.

\section{References}

[1] T.A. Wynn, Fibrotic disease and the $T(H) 1 / T(H) 2$ paradigm, Nat Rev Immunol 4(8) (2004) 583-94.

[2] P.C. Lara, D. Macias-Verde, J. Burgos-Burgos, Age-induced NLRP3 Inflammasome Overactivation Increases Lethality of SARS-CoV-2 Pneumonia in Elderly Patients, Aging Dis 11(4) (2020) 756-762.

[3] S.M. Williams, L. Golden-Mason, B.S. Ferguson, K.B. Schuetze, M.A. Cavasin, K. DemosDavies, M.E. Yeager, K.R. Stenmark, T.A. McKinsey, Class I HDACs regulate angiotensin IIdependent cardiac fibrosis via fibroblasts and circulating fibrocytes, J Mol Cell Cardiol 67 (2014) 112-25.

[4] V. Grimaldi, M.R. De Pascale, A. Zullo, A. Soricelli, T. Infante, F.P. Mancini, C. Napoli, Evidence of epigenetic tags in cardiac fibrosis, J Cardiol 69(2) (2017) 401-408.

[5] D. Fan, A. Takawale, J. Lee, Z. Kassiri, Cardiac fibroblasts, fibrosis and extracellular matrix remodeling in heart disease, Fibrogenesis Tissue Repair 5(1) (2012) 15.

[6] A. Kundaje, W. Meuleman, J. Ernst, M. Bilenky, A. Yen, A. Heravi-Moussavi, P. Kheradpour, Z. Zhang, J. Wang, M.J. Ziller, V. Amin, J.W. Whitaker, M.D. Schultz, L.D. Ward, A. Sarkar, G. Quon, R.S. Sandstrom, M.L. Eaton, Y.C. Wu, A.R. Pfenning, X. Wang, M. Claussnitzer, Y. Liu, C. Coarfa, R.A. Harris, N. Shoresh, C.B. Epstein, E. Gjoneska, D. Leung, W. Xie, R.D. Hawkins, R. Lister, C. Hong, P. Gascard, A.J. Mungall, R. Moore, E. Chuah, A. Tam, T.K. Canfield, R.S. Hansen, R. Kaul, P.J. Sabo, M.S. Bansal, A. Carles, J.R. Dixon, K.H. Farh, S. Feizi, R. Karlic, A.R. Kim, A. Kulkarni, D. Li, R. Lowdon, G. Elliott, T.R. Mercer, S.J. Neph, V. Onuchic, P. Polak, N. Rajagopal, P. Ray, R.C. Sallari, K.T. Siebenthall, N.A. Sinnott-Armstrong, M. Stevens, R.E. Thurman, J. Wu, B. Zhang, X. Zhou, A.E. Beaudet, L.A. Boyer, P.L. De Jager, P.J. Farnham, S.J. Fisher, D. Haussler, S.J. Jones, W. Li, M.A. Marra, M.T. McManus, S. Sunyaev, J.A. Thomson, T.D. Tlsty, L.H. Tsai, W. Wang, R.A. Waterland, M.Q. Zhang, L.H. Chadwick, B.E. Bernstein, J.F. Costello, J.R. Ecker, M. Hirst, A. Meissner, A. Milosavljevic, B. Ren, J.A. Stamatoyannopoulos, T. Wang, M. Kellis, Integrative analysis of 111 reference human epigenomes, Nature 518(7539) (2015) 317-30. 
[7] R. Papait, S. Serio, G. Condorelli, Role of the Epigenome in Heart Failure, Physiol Rev 100(4) (2020) 1753-1777.

[8] M. Ehrlich, DNA hypermethylation in disease: mechanisms and clinical relevance, Epigenetics 14(12) (2019) 1141-1163.

[9] M.B. Felisbino, T.A. McKinsey, Epigenetics in Cardiac Fibrosis: Emphasis on Inflammation and Fibroblast Activation, JACC Basic Transl Sci 3(5) (2018) 704-715.

[10] K. Al-Hasani, P. Mathiyalagan, A. El-Osta, Epigenetics, cardiovascular disease, and cellular reprogramming, J Mol Cell Cardiol 128 (2019) 129-133.

[11] M. Alexanian, A. Padmanabhan, T.A. McKinsey, S.M. Haldar, Epigenetic therapies in heart failure, J Mol Cell Cardiol 130 (2019) 197-204.

[12] H. Tao, Z.Y. Song, X.S. Ding, J.J. Yang, K.H. Shi, J. Li, Epigenetic signatures in cardiac fibrosis, special emphasis on DNA methylation and histone modification, Heart Fail Rev 23(5) (2018) 789-799.

[13] X. Lyu, M. Hu, J. Peng, X. Zhang, Y.Y. Sanders, HDAC inhibitors as antifibrotic drugs in cardiac and pulmonary fibrosis, Ther Adv Chronic Dis 10 (2019) 2040622319862697.

[14] M. Wei, L. Wang, T. Wu, J. Xi, Y. Han, X. Yang, D. Zhang, Q. Fang, B. Tang, NLRP3 Activation Was Regulated by DNA Methylation Modification during Mycobacterium tuberculosis Infection, Biomed Res Int 2016 (2016) 4323281.

[15] R. Vento-Tormo, D. Alvarez-Errico, A. Garcia-Gomez, J. Hernandez-Rodriguez, S. Bujan, M. Basagana, M. Mendez, J. Yague, M. Juan, J.I. Arostegui, E. Ballestar, DNA demethylation of inflammasome-associated genes is enhanced in patients with cryopyrin-associated periodic syndromes, J Allergy Clin Immunol 139(1) (2017) 202-211 e6.

[16] L.E. Dorn, J.M. Petrosino, P. Wright, F. Accornero, CTGF/CCN2 is an autocrine regulator of cardiac fibrosis, J Mol Cell Cardiol 121 (2018) 205-211.

[17] J.G. Travers, F.A. Kamal, J. Robbins, K.E. Yutzey, B.C. Blaxall, Cardiac Fibrosis: The Fibroblast Awakens, Circ Res 118(6) (2016) 1021-40.

[18] A.A. Pinar, T.E. Scott, B.M. Huuskes, F.E. Tapia Caceres, B.K. Kemp-Harper, C.S. Samuel, Targeting the NLRP3 inflammasome to treat cardiovascular fibrosis, Pharmacol Ther 209 (2020) 107511.

[19] S. Vukelic, K.K. Griendling, Angiotensin II, from vasoconstrictor to growth factor: a paradigm shift, Circ Res 114(5) (2014) 754-7.

[20] B. Poudel, P. Gurung, An update on cell intrinsic negative regulators of the NLRP3 inflammasome, J Leukoc Biol (2018).

[21] A.A.A. Alyaseer, M.H.S. de Lima, T.T. Braga, The Role of NLRP3 Inflammasome Activation in the Epithelial to Mesenchymal Transition Process During the Fibrosis, Front Immunol 11 (2020) 883.

[22] B. Chaqour, Caught between a "Rho" and a hard place: are CCN1/CYR61 and CCN2/CTGF the arbiters of microvascular stiffness?, J Cell Commun Signal 14(1) (2020) 21-29.

[23] Y. Ramazani, N. Knops, M.A. Elmonem, T.Q. Nguyen, F.O. Arcolino, L. van den Heuvel, E. Levtchenko, D. Kuypers, R. Goldschmeding, Connective tissue growth factor (CTGF) from basics to clinics, Matrix Biol 68-69 (2018) 44-66.

[24] M. Labrie, Y. St-Pierre, Epigenetic regulation of mmp-9 gene expression, Cell Mol Life Sci 70(17) (2013) 3109-24.

[25] N. Glezeva, B. Moran, P. Collier, C.S. Moravec, D. Phelan, E. Donnellan, A. Russell-Hallinan, D.P. O'Connor, W.M. Gallagher, J. Gallagher, K. McDonald, M. Ledwidge, J. Baugh, S. Das, C.J. Watson, Targeted DNA Methylation Profiling of Human Cardiac Tissue Reveals Novel Epigenetic 
Traits and Gene Deregulation Across Different Heart Failure Patient Subtypes, Circ Heart Fail 12(3) (2019) e005765.

[26] J. He, M. Qin, Y. Chen, Z. Hu, F. Xie, L. Ye, T. Hui, Epigenetic regulation of matrix metalloproteinases in inflammatory diseases: a narrative review, Cell Biosci 10 (2020) 86.

[27] G. Poli, C. Fabi, M.M. Bellet, C. Costantini, L. Nunziangeli, L. Romani, S. Brancorsini, Epigenetic Mechanisms of Inflammasome Regulation, Int J Mol Sci 21(16) (2020).

[28] Y. Nibu, S. Takahashi, K. Tanimoto, K. Murakami, A. Fukamizu, Identification of cell typedependent enhancer core element located in the 3'-downstream region of the human angiotensinogen gene, J Biol Chem 269(46) (1994) 28598-605.

[29] L.Q. Fan, D.O. Hardy, J.F. Catterall, J. Zhao, S.X. Li, Identification and characterization of an androgen-responsive Kap promoter enhancer located in the intron II region of human angiotensinogen gene, J Steroid Biochem Mol Biol 119(3-5) (2010) 135-40.

[30] The_GTEx_Consortium, Human genomics. The genotype-tissue expression (GTEx) pilot analysis: Multitissue gene regulation in humans, Science 348(6235) (2015) 648-60.

[31] K.R. Rosenbloom, J. Armstrong, G.P. Barber, J. Casper, H. Clawson, M. Diekhans, T.R. Dreszer, P.A. Fujita, L. Guruvadoo, M. Haeussler, R.A. Harte, S. Heitner, G. Hickey, A.S. Hinrichs, R. Hubley, D. Karolchik, K. Learned, B.T. Lee, C.H. Li, K.H. Miga, N. Nguyen, B. Paten, B.J. Raney, A.F. Smit, M.L. Speir, A.S. Zweig, D. Haussler, R.M. Kuhn, W.J. Kent, The UCSC Genome Browser database: 2015 update, Nucleic Acids Res 43(Database issue) (2015) D670-81.

[32] K. Tsumagari, C. Baribault, J. Terragni, K.E. Varley, J. Gertz, S. Pradhan, M. Badoo, C.M. Crain, L. Song, G.E. Crawford, R.M. Myers, M. Lacey, M. Ehrlich, Early de novo DNA methylation and prolonged demethylation in the muscle lineage, Epigenetics 8(3) (2013) 317-32.

[33] S. Zaina, H. Heyn, F.J. Carmona, N. Varol, S. Sayols, E. Condom, J. Ramirez-Ruz, A. Gomez, I. Goncalves, S. Moran, M. Esteller, DNA methylation map of human atherosclerosis, Circ Cardiovasc Genet 7(5) (2014) 692-700.

[34] M. Lacey, C. Baribault, K.C. Ehrlich, M. Ehrlich, Atherosclerosis-associated differentially methylated regions can reflect the disease phenotype and are often at enhancers, Atherosclerosis 280 (2019) 183-191.

[35] A. Khan, X. Zhang, dbSUPER: a database of super-enhancers in mouse and human genome, Nucleic Acids Res 44(D1) (2016) D164-71.

[36] F.G. Bauernfeind, G. Horvath, A. Stutz, E.S. Alnemri, K. MacDonald, D. Speert, T. FernandesAlnemri, J. Wu, B.G. Monks, K.A. Fitzgerald, V. Hornung, E. Latz, Cutting edge: NF-kappaB activating pattern recognition and cytokine receptors license NLRP3 inflammasome activation by regulating NLRP3 expression, J Immunol 183(2) (2009) 787-91.

[37] Y. Wang, X. Liu, H. Shi, Y. Yu, Y. Yu, M. Li, R. Chen, NLRP3 inflammasome, an immuneinflammatory target in pathogenesis and treatment of cardiovascular diseases, Clin Transl Med 10(1) (2020) 91-106.

[38] W.A. Whyte, D.A. Orlando, D. Hnisz, B.J. Abraham, C.Y. Lin, M.H. Kagey, P.B. Rahl, T.I. Lee, R.A. Young, Master transcription factors and mediator establish super-enhancers at key cell identity genes, Cell 153(2) (2013) 307-19.

[39] G. Guarda, M. Zenger, A.S. Yazdi, K. Schroder, I. Ferrero, P. Menu, A. Tardivel, C. Mattmann, J. Tschopp, Differential expression of NLRP3 among hematopoietic cells, J Immunol 186(4) (2011) 2529-34.

[40] M. Bruchard, C. Rebe, V. Derangere, D. Togbe, B. Ryffel, R. Boidot, E. Humblin, A. Hamman, F. Chalmin, H. Berger, A. Chevriaux, E. Limagne, L. Apetoh, F. Vegran, F. Ghiringhelli, The 
receptor NLRP3 is a transcriptional regulator of TH2 differentiation, Nat Immunol 16(8) (2015) 859-70.

[41] J. Wang, J. Zhuang, S. Iyer, X.Y. Lin, M.C. Greven, B.H. Kim, J. Moore, B.G. Pierce, X. Dong, D. Virgil, E. Birney, J.H. Hung, Z. Weng, Factorbook.org: a Wiki-based database for transcription factor-binding data generated by the ENCODE consortium, Nucleic Acids Res 41(Database issue) (2013) D171-6.

[42] V.K. Ponnaluri, K.C. Ehrlich, G. Zhang, M. Lacey, D. Johnston, S. Pradhan, M. Ehrlich, Association of 5-hydroxymethylation and 5-methylation of DNA cytosine with tissue-specific gene expression, Epigenetics 12(2) (2017) 123-138.

[43] M. Haneklaus, M. Gerlic, M. Kurowska-Stolarska, A.A. Rainey, D. Pich, I.B. McInnes, W. Hammerschmidt, L.A. O'Neill, S.L. Masters, Cutting edge: miR-223 and EBV miR-BART15 regulate the NLRP3 inflammasome and IL-1beta production, J Immunol 189(8) (2012) 3795-9.

[44] V. Sartorelli, S.M. Lauberth, Enhancer RNAs are an important regulatory layer of the epigenome, Nat Struct Mol Biol 27(6) (2020) 521-528.

[45] S.S. Rao, M.H. Huntley, N.C. Durand, E.K. Stamenova, I.D. Bochkov, J.T. Robinson, A.L. Sanborn, I. Machol, A.D. Omer, E.S. Lander, E.L. Aiden, A 3D map of the human genome at kilobase resolution reveals principles of chromatin looping, Cell 159(7) (2014) 1665-80.

[46] S. Wang, J. Chen, S.P. Garcia, X. Liang, F. Zhang, P. Yan, H. Yu, W. Wei, Z. Li, J. Wang, H. Le, Z. Han, X. Luo, D.S. Day, S.M. Stevens, Y. Zhang, P.J. Park, Z.J. Liu, K. Sun, G.C. Yuan, W.T. Pu, B. Zhang, A dynamic and integrated epigenetic program at distal regions orchestrates transcriptional responses to VEGFA, Genome Res 29(2) (2019) 193-207.

[47] H. Yang, Y. Cao, J. Zhang, Y. Liang, X. Su, C. Zhang, H. Liu, X. Han, L. Ge, Z. Fan, DLX5 and HOXC8 enhance the chondrogenic differentiation potential of stem cells from apical papilla via LINC01013, Stem Cell Res Ther 11(1) (2020) 271.

[48] I.H. Chung, P.H. Lu, Y.H. Lin, M.M. Tsai, Y.W. Lin, C.T. Yeh, K.H. Lin, The long non-coding RNA LINC01013 enhances invasion of human anaplastic large-cell lymphoma, Sci Rep 7(1) (2017) 295.

[49] S.G. Boaru, E. Borkham-Kamphorst, E. Van de Leur, E. Lehnen, C. Liedtke, R. Weiskirchen, NLRP3 inflammasome expression is driven by NF-kappaB in cultured hepatocytes, Biochem Biophys Res Commun 458(3) (2015) 700-706.

[50] A. Kumar, N. Nallabelli, U. Sharma, N. Kumari, S.K. Singh, N. Kakkar, R. Prasad, In vitro evidence of NLRP3 inflammasome regulation by histone demethylase LSD2 in renal cancer: a pilot study, Mol Biol Rep (2020).

[51] Z. Ungvari, M.N. Valcarcel-Ares, S. Tarantini, A. Yabluchanskiy, G.A. Fulop, T. Kiss, A. Csiszar, Connective tissue growth factor (CTGF) in age-related vascular pathologies, Geroscience 39(5-6) (2017) 491-498.

[52] W.B. Wang, H.P. Li, J. Yan, F. Zhuang, M. Bao, J.T. Liu, Y.X. Qi, Y. Han, CTGF regulates cyclic stretch-induced vascular smooth muscle cell proliferation via microRNA-19b-3p, Exp Cell Res 376(1) (2019) 77-85.

[53] A.R. Brasier, J.E. Tate, D. Ron, J.F. Habener, Multiple cis-acting DNA regulatory elements mediate hepatic angiotensinogen gene expression, Mol Endocrinol 3(6) (1989) 1022-34.

[54] S. Ounzain, T. Pedrazzini, Super-enhancer Incs to cardiovascular development and disease, Biochim Biophys Acta 1863(7 Pt B) (2016) 1953-60.

[55] A.R. Brasier, J. Zhou, Validation of the epigenetic reader bromodomain-containing protein 4 (BRD4) as a therapeutic target for treatment of airway remodeling, Drug Discov Today 25(1) (2020) 126-132. 
[56] L.A. O'Neill, F.J. Sheedy, C.E. McCoy, MicroRNAs: the fine-tuners of Toll-like receptor signalling, Nat Rev Immunol 11(3) (2011) 163-75.

[57] M. de Kerckhove, K. Tanaka, T. Umehara, M. Okamoto, S. Kanematsu, H. Hayashi, H. Yano, S. Nishiura, S. Tooyama, Y. Matsubayashi, T. Komatsu, S. Park, Y. Okada, R. Takahashi, Y. Kawano, T. Hanawa, K. Iwasaki, T. Nozaki, H. Torigoe, K. Ikematsu, Y. Suzuki, K. Tanaka, P. Martin, I. Shimokawa, R. Mori, Targeting miR-223 in neutrophils enhances the clearance of Staphylococcus aureus in infected wounds, EMBO Mol Med 10(10) (2018).

[58] L. Vian, M. Di Carlo, E. Pelosi, F. Fazi, S. Santoro, A.M. Cerio, A. Boe, V. Rotilio, M. Billi, S. Racanicchi, U. Testa, F. Grignani, C. Nervi, Transcriptional fine-tuning of microRNA-223 levels directs lineage choice of human hematopoietic progenitors, Cell Death Differ 21(2) (2014) 290301.

[59] M.P. Roffel, K.R. Bracke, I.H. Heijink, T. Maes, miR-223: A Key Regulator in the Innate Immune Response in Asthma and COPD, Front Med (Lausanne) 7 (2020) 196.

[60] M. Haneklaus, J.D. O'Neil, A.R. Clark, S.L. Masters, L.A.J. O'Neill, The RNA-binding protein Tristetraprolin (TTP) is a critical negative regulator of the NLRP3 inflammasome, J Biol Chem 292(17) (2017) 6869-6881.

[61] K. Wang, B. Long, F. Liu, J.X. Wang, C.Y. Liu, B. Zhao, L.Y. Zhou, T. Sun, M. Wang, T. Yu, Y. Gong, J. Liu, Y.H. Dong, N. Li, P.F. Li, A circular RNA protects the heart from pathological hypertrophy and heart failure by targeting miR-223, Eur Heart J 37(33) (2016) 2602-11.

[62] K.V. Swanson, M. Deng, J.P. Ting, The NLRP3 inflammasome: molecular activation and regulation to therapeutics, Nat Rev Immunol 19(8) (2019) 477-489.

[63] F. Yu, C.W. Chou, C.C. Chen, TNF-alpha suppressed TGF-beta-induced CTGF expression by switching the binding preference of p300 from Smad4 to p65, Cell Signal 21(6) (2009) 867-72. 